\title{
Monitoring seasonal and diurnal changes in photosynthetic pigments with automated PRI and NDVI sensors
}

\author{
J. A. Gamon ${ }^{1,2}$, O. Kovalchuck ${ }^{1}$, C. Y. S. Wong ${ }^{1, a}$, A. Harris ${ }^{3}$, and S. R. Garrity ${ }^{4}$ \\ ${ }^{1}$ Department of Earth \& Atmospheric Sciences, 1-26 Earth Sciences Building, University of Alberta, Edmonton, Alberta, \\ T6G 2E3, Canada \\ ${ }^{2}$ Department of Biological Sciences, University of Alberta, Edmonton, Alberta, Canada \\ ${ }^{3}$ Geography, School of Environment, Education and Development, University of Manchester, Manchester, UK \\ ${ }^{4}$ Decagon Devices, Inc., 2365 NE Hopkins Court, Pullman, Washington 99163, USA \\ ${ }^{a}$ current address: Department of Biology, 3359 Mississauga Road, University of Toronto Mississauga, Mississauga, L5L 1C6, \\ Canada
}

Correspondence to: J. A. Gamon (jgamon@gmail.com)

Received: 27 November 2014 - Published in Biogeosciences Discuss.: 10 February 2015

Revised: 29 May 2015 - Accepted: 16 June 2015 - Published: 14 July 2015

\begin{abstract}
The vegetation indices normalized difference vegetation index (NDVI) and photochemical reflectance index (PRI) provide indicators of pigmentation and photosynthetic activity that can be used to model photosynthesis from remote sensing with the light-use-efficiency model. To help develop and validate this approach, reliable proximal NDVI and PRI sensors have been needed. We tested new NDVI and PRI sensors, "spectral reflectance sensors" (SRS sensors; recently developed by Decagon Devices, during spring activation of photosynthetic activity in evergreen and deciduous stands. We also evaluated two methods of sensor cross-calibration - one that considered sky conditions (cloud cover) at midday only, and another that also considered diurnal sun angle effects. Cross-calibration clearly affected sensor agreement with independent measurements, with the best method dependent upon the study aim and time frame (seasonal vs. diurnal). The seasonal patterns of NDVI and PRI differed for evergreen and deciduous species, demonstrating the complementary nature of these two indices. Over the spring season, PRI was most strongly influenced by changing chlorophyll : carotenoid pool sizes, while over the diurnal timescale, PRI was most affected by the xanthophyll cycle epoxidation state. This finding demonstrates that the SRS PRI sensors can resolve different processes affecting PRI over different timescales. The advent of small, inexpensive, automated PRI and NDVI sensors offers new ways to explore environmental and physiological constraints on photosynthe-
\end{abstract}

sis, and may be particularly well suited for use at flux tower sites. Wider application of automated sensors could lead to improved integration of flux and remote sensing approaches for studying photosynthetic carbon uptake, and could help define the concept of contrasting vegetation optical types.

\section{Introduction}

The photochemical reflectance index (PRI) was originally derived as a measure of xanthophyll cycle activity determined using proximal remote sensing of leaves and canopies on a diurnal timescale (Gamon et al., 1992, 1997). In this context, the xanthophyll cycle is a facultative response that changes readily as a means of dissipating extra light energy non-destructively (Demmig-Adams and Adams, 1992). Because this xanthophyll response reflects changing light energy distribution within the photosynthetic system, it can provide a useful measure of short-term changes in photosynthetic light-use efficiency, typically expressed as the photosynthetic rate normalized by the incident or absorbed photosynthetically active radiation (Gamon et al., 1992, 1997; Peñuelas et al., 1995). However, studies at larger scales spanning species or seasons often reveal a different story; when sampled at these larger spatial or temporal scales, PRI is strongly influenced by evolving chlorophyll : carotenoid ratios (Sims and Gamon, 2002; Stylinski et al., 2002; Filella 
et al., 2009; Garrity et al., 2011; Gamon and Berry, 2012; Porcar-Castell et al., 2012; Wong and Gamon, 2015a) Unlike the diurnal xanthophyll cycle activity, these pigment pool size adjustments comprise a constitutive response to chronic stress, ontogeny, or phenology, determined by more slowly changing physiological states in response to time-integrated environmental conditions. For example, during seasonal transitions from a dormant to an active growth phase, evergreen plants adjust their chlorophyll : carotenoid ratios over many weeks in response to changing temperatures (Adams et al., 2002), and this adjustment can be readily detected by PRI (Stylinski et al., 2002; Filella et al., 2009; Porcar-Castell et al., 2012; Wong and Gamon, 2015a). Both the facultative and constitutive PRI responses are strongly correlated with photosynthetic activity but over different timescales and using different mechanisms, both of which involve photoprotective carotenoid pigments.

The normalized difference vegetation index (NDVI) was developed as a measure of vegetation greenness. Typically, it is used to evaluate seasonal phenology or productivity of vegetation as it changes gradually with the growth and senescence of vegetation (Gamon et al., 1995). NDVI is also a common product of many satellite sensors and is widely used for tracking vegetation phenology, and mapping potential photosynthetic activity or productivity (Defries and Townshend, 1994; Running et al., 2004). However, NDVI-based approaches often miss more subtle, short-term responses to stress that can determine how much of the photosynthetic potential is actually realized, particularly for species showing few structural responses to stress. For example, in annual or deciduous canopies, NDVI is highly correlated with morphological changes (green biomass or leaf area index) that affect seasonally changing photosynthetic capacity. In evergreens, where canopy structure is relatively stable over the year, NDVI changes little with season and fails to detect the onset and cessation of photosynthesis early and late in the growing season (Gamon et al., 1995). Thus, while NDVI is well suited for detecting photosynthetic potential defined by light absorption and canopy structure, it misses many of the more subtle photosynthetic dynamics arising from alterations in physiological activity (e.g., photosynthetic downregulation during short-term stress). For this, PRI is often a useful counterpart.

When combined, NDVI and PRI can provide complementary information regarding photosynthetic activity. Together, NDVI and PRI can be used to estimate photosynthetic rate, typically using a light-use efficiency model, with NDVI providing a means to estimate light absorption by green vegetation and PRI providing a measure of the efficiency with which that absorbed light is converted to fixed carbon (Gamon and Qiu, 1999; Gamon et al., 2001). When integrated over time (typically a growing season), the photosynthetic rate estimated from vegetation indices can provide a good measure of net primary production, NPP (Goward et al., 1985). Singly or in combination, these two indices provide a means to estimate photosynthetic phenology and activity, with the exact use depending upon the particular application and time frame.

Originally, most field studies employing these vegetation indices were conducted with portable spectrometers capable of measuring reflectance in many wavebands simultaneously. While these instruments represent the "gold standard" for field reflectance measurements, many are bulky, expensive, and are not well suited to long-term, automated deployment in the field without additional modifications for unattended use (e.g., Hilker et al., 2007; Rossini et al., 2012; Drolet et al., 2014). Given the relationship between NDVI, PRI, and photosynthetic carbon uptake or NPP, there is a growing interest in monitoring these vegetation indices within the footprint of flux towers that measure the gas exchange of whole ecosystems. PRI measurements are particularly problematic because they require high instrument sensitivity (signal-tonoise ratio) and stability (Castro-Esau et al., 2006), and because of the many factors that can confound PRI interpretation (Barton and North, 2001). Furthermore, automated sensors typically require dual-view configurations with up- and down-facing detectors that must be well matched spectrally and radiometrically if they are to be comparable (Harris et al., 2014). To address the need for automated field measurements, new small and inexpensive optical sensors are emerging that can monitor dynamic vegetation indices such as PRI and NDVI (Garrity et al., 2010; Ryu et al., 2010; Eklundh et al., 2011; Harris et al., 2014). These automated sensors need to be tested and evaluated against field spectrometers, particularly if data are to be compared across sites and research deployments employing different instruments.

The goals of this study were to (1) develop field measurement protocols for inexpensive, automated sensors; (2) compare the NDVI and PRI measured by these sensors to independent spectrometer measurements; (3) explore the complementary behavior of PRI and NDVI in deciduous and evergreen canopies; and (4) evaluate whether the PRI signals obtained in this way can distinguish the facultative and constitutive pigment responses. Because of the challenges with obtaining meaningful PRI measurements, our particular focus was on evaluating PRI sensors (SRS, Decagon Devices Inc., Pullman, WA, USA), in part by comparing them to the response of established "industry-standard" field spectrometers (UniSpec and UniSpec DC, PP Systems, Amesbury, MA, USA), but also by characterizing their responses to environmental and physiological factors known to influence PRI. We compared the results of the new PRI sensors to leaf pigments measured over different timescales in evergreen conifers as a means of evaluating the facultative and constitutive components of the PRI signals. 


\section{Methods}

\subsection{Study design and plant culture}

Over a 2-month period (03 May to 21 June 2013), automated SRS sensors were used to monitor two single-species stands, and these data were compared to measurements from field spectrometers. This 2-month period covered the spring transition from late winter to early summer. The study was conducted on a rooftop common garden located at the University of Alberta campus, Edmonton, Alberta, Canada. Two plant species were used: lodgepole pine (Pinus contorta, an evergreen conifer) and aspen (Populus tremuloides, a winterdeciduous tree). During the study period, the aspen leaves emerged from dormant buds and the pine species recovered its photosynthetic activity following winter downregulation. We used midday measurements to focus on the seasonal transition. On 25 July 2013, we performed a diurnal study to detect variations in PRI and NDVI with time of day or sky conditions, and to understand the effect of sensor crosscalibration methods on the temporal patterns of these sensor index values. We compared PRI measurements from both seasonal and diurnal studies to changing pigment composition to evaluate the facultative and constitutive pigment contributions to the PRI signal.

The pine seedlings were approximately 4 years old and the aspen was approximately 3 years old at the time the study began (2013). Plants were potted in large, $6.23 \mathrm{~L}$ pots, using a $1: 2$ mix of sandy loam and commercial potting soil (Sunshine Mix 4, Sun Gro Horticulture, Agawam, MA, USA) supplemented with slow release fertilizer (Nutricote 14-1414, Sun Gro Horticulture, Agawam, MA, USA). All plants were fertilized periodically and watered regularly to avoid nutrient and drought stress during the measurement period. Potted seedlings were arranged into $1.5 \times 1.5 \mathrm{~m}$ arrays comprising two synthetic plant stands, providing closed canopy monocultures for viewing by the sensors.

\subsection{Optical measurements}

The automated sensors consisted of five PRI sensors and five NDVI spectral reflectance sensors (SRS sensors, Decagon Inc, Pullman, WA, USA). These were early prototypes of the SRS sensors currently available from Decagon Devices Inc. The detectors of the prototype PRI sensors were photodiodes paired with interference filters centered at the 532 and $570 \mathrm{~nm}$ PRI wavelengths, similar to those used by Garrity et al. (2010). The interference filters have a bandpass of $10 \mathrm{~nm}$ at full width at half maximum (FWHM). Following Ryu et al. (2010), prototype NDVI sensors used light emitting diodes (LEDs). LEDs had peak sensitivity at 630 and $800 \mathrm{~nm}$ with bandpass widths of 50 and $40 \mathrm{~nm}$, respectively. Note that since our study was completed, the manufacturer has changed the NDVI sensor to be based on a photodiode design.
Of the five PRI ("P") sensors, three were downwardlooking sensors ("Pr1", "Pr2", and "Pr3", where "r" indicates radiance), with a field of view (FOV) of approximately $20^{\circ}$ full angle, and two were upward-looking hemispherical sensors ("Pi4" and "Pi5", where "i" indicates irradiance), with a FOV of approximately $180^{\circ}$ (full angle). Of the five NDVI ("N") sensors, three were downward-looking sensors ("Nr1", "Nr2", and "Nr3", where "r" indicates radiance) and two were upward-looking sensors ("Ni4" and "Ni5", where "i" indicates irradiance). The upward-looking hemispherical sensors provided reference values of sky irradiance against which we normalized the downward-looking sensor values of canopy radiance using a cross-calibration procedure described in Sect. 2.3.

The 10 SRS sensors were positioned above the two plant stands at a height of approximately $2 \mathrm{~m}$ above the ground. Due to differences in canopy height, the exact distance between the sensors and the tops of the canopies varied as follows: $45 \mathrm{~cm}$ for lodgepole pine and $50 \mathrm{~cm}$ for aspen. In all cases, downward-looking canopy radiance sensors were positioned over the center of the plant stands to avoid possible edge effects. The upward-looking sensors were mounted above the middle of the canopies to monitor sky irradiance.

Each sensor was logged every $5 \mathrm{~s}$ and expressed as $1 \mathrm{~min}$ averages by a data logger (CR1000, Campbell Scientific, Logan, UT, USA). To calculate reflectance, data from each of the three downward-looking PRI and NDVI (radiance) sensors were compared to the average of the coincident measurements made by the two upward-looking PRI and NDVI (irradiance) sensors, respectively. For each waveband, uncorrected reflectance was first calculated by dividing the radiance by the irradiance values:

$\frac{\operatorname{Pr}_{532 \mathrm{~nm}}}{\mathrm{Pi}_{532 \mathrm{~nm}}}$,
$\frac{\mathrm{Pr}_{570 \mathrm{~nm}}}{\mathrm{Pi}_{570 \mathrm{~nm}}}$,
$\frac{\mathrm{Nr}_{630 \mathrm{~nm}}}{\mathrm{Ni}_{630 \mathrm{~nm}}}$,
$\frac{\mathrm{Nr}_{800 \mathrm{~nm}}}{\mathrm{Ni}_{800 \mathrm{~nm}}}$.

These uncorrected reflectance values $(\mathrm{Pr} / \mathrm{Pi}$ and $\mathrm{Nr} / \mathrm{Ni})$ calculated for each waveband were then used to calculate uncorrected PRI (using Eq. 2) and NDVI (using Eq. 3), respectively.

$$
\begin{aligned}
& \mathrm{PRI}=\frac{\operatorname{Pr} / \mathrm{Pi}_{532 \mathrm{~nm}}-\mathrm{Pr} / \mathrm{Pi}_{570 \mathrm{~nm}}}{\mathrm{Pr} / \mathrm{Pi}_{532 \mathrm{~nm}}+\mathrm{Pr} / \mathrm{Pi}_{570 \mathrm{~nm}}}, \\
& \mathrm{NDVI}=\frac{\mathrm{Nr} / \mathrm{Ni}_{800 \mathrm{~nm}}-\mathrm{Nr} / \mathrm{Ni}_{630 \mathrm{~nm}}}{\mathrm{Nr} / \mathrm{Ni}_{800 \mathrm{~nm}}+\mathrm{Nr} / \mathrm{Ni}_{630 \mathrm{~nm}}} .
\end{aligned}
$$

Each uncorrected reflectance measurement was further modified by a sensor cross-calibration coefficient (see below), yielding corrected reflectance and allowing us to evaluate the effect of this coefficient on the PRI or NDVI signals. 


\subsection{Sensor cross-calibration}

Previous studies (Gamon et al., 2006) have illustrated the need for cross-calibration to properly match radiance and irradiance sensor outputs when calculating reflectance from dual-detector (radiance and irradiance) optical sensors. This need arises from the different sensor responses and foreoptics, which must be normalized to yield correct reflectance and index values. In this study, a similar cross-calibration was performed by correcting each radiance sensor against the matching pair of irradiance sensors used for raw reflectance calculation. The cross-calibration procedure involved the insertion of a $25 \times 25 \mathrm{~cm}, 99 \%$ reflective white panel (Spectralon, Labsphere Inc., North Sutton, NH, USA) covering the FOV of each downward-looking sensor, while the upwardlooking sensors sampled sky irradiance. For each crosscalibration, the panel was held under the downward-looking sensors at an approximate distance of $20 \mathrm{~cm}$ for 5 consecutive minutes and the measurements made during this period were averaged to obtain a single cross-calibration for that sensor at that time for those particular sky conditions (cloud cover and sun angle). This procedure yielded a cross-calibration ratio ("cross-calibration"), expressed as $\mathrm{Pr}_{\text {panel }} / \mathrm{Pi}_{\text {sky }}$ and $\mathrm{Nr}_{\text {panel }} / \mathrm{Ni}_{\text {sky }}$ for each band and sensor pair.

To explore the effect of cloud cover on seasonally changing indices, midday measurements from 11:00 to 15:00 local time (LT) (where solar noon was approximately 13:30 LT) were used. These midday cross-calibration ratios were plotted as a function of cloud cover (expressed as the ratio of sun visibility) in order to evaluate the relationship between the cross-calibration ratios and cloud cover. The sun visibility ratio was calculated by comparing the actual photosynthetic photon flux density (i.e. PAR irradiance) measured on several dates $(09,15,28$, and 30 May and 04 and 05 June 2013) to a modeled PPFD assuming perfectly sunny conditions. Actual PPFD was sampled with a quantum sensor (model S-LIAM003, Onset Computer Corporation, Bourne, MA, USA) and data logger (HOBO U30, Onset Computer Corporation, Bourne, MA, USA). Modeled PPFD was calculated with the Solar Radiation Calculator (SolRad), using the RyanStolzenbach modeled global solar radiation on a horizontal surface (http://www.ecy.wa.gov/programs/eap/models.html). Theoretically, the resulting sun visibility ratio values varied between zero (complete darkness) and one (clear, sunny skies), with values falling in between indicating varying degrees of cloudiness.

Using Eqs. (4) and (5), midday cross-calibration ratios were calculated for a range of sun visibility conditions encountered on typical sunny and cloudy days. The resulting functions were used to generate empirical equations for each sensor and waveband, normalizing the uncorrected reflectance values for each channel by their cross-calibration ratios.

$$
\begin{aligned}
& \mathrm{Pr} / \mathrm{Pi}_{\text {corrected }}=\frac{\mathrm{Pr}_{\text {target }} / \mathrm{Pi}_{\text {sky }}}{\mathrm{Pr}_{\text {panel }} / \mathrm{Pi}_{\text {sky }}} \\
& =\frac{\mathrm{Pr}_{\text {target }} / \mathrm{Pi}_{\text {sky }}}{\text { Crosscalibration ratio }}, \\
& \mathrm{Nr} / \mathrm{Ni}_{\text {corrected }}=\frac{\mathrm{Nr}_{\text {target }} / \mathrm{Ni}_{\text {sky }}}{\mathrm{Nr}_{\text {panel }} / \mathrm{Ni}_{\text {sky }}} \\
& =\frac{\mathrm{Nr}_{\text {target }} / \mathrm{Ni}_{\text {sky }}}{\text { Crosscalibration ratio }},
\end{aligned}
$$

where the subscripts "target", "panel" and "sky" indicate radiance measurements of the canopy, radiance measurements of the white panel and irradiance measurements of the sky, respectively. The corrected signal was then used to calculate a midday-corrected PRI (Eq. 6) and NDVI (Eq. 7) for evaluation of seasonal trends.

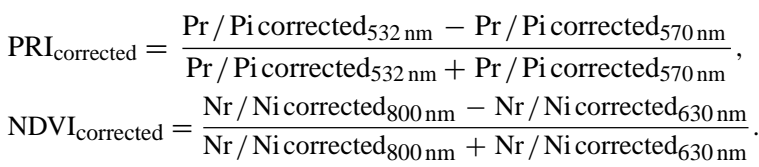

Diurnal cross-calibration proved more challenging because it was affected by sun angle and cloud cover, and both changed in complex ways over the course of a typical day. To explore the effect of these diurnal changes, the crosscalibration of each Decagon SRS was performed approximately once an hour from 06:30 to 18:30 LT. Consequently, corrected reflectance and indices for diurnal experiments used an empirical cross-calibration derived for each sensor using the hourly cross-calibration ratios collected closest in time to that sample, incorporating the combined effects of both sun angle and sky conditions on the cross-calibration. These empirical, whole-day corrections were compared to corrections using midday cross-calibrations (Eqs. 6 and 7) and to "raw" sensor index values uncorrected by crosscalibration (Eqs. 2 and 3). For plotting, the corrected $1 \mathrm{~min}$ PRI and NDVI samples were averaged over $15 \mathrm{~min}$ (for diurnal studies) or $1 \mathrm{~h}$ (for seasonal studies), creating a single value for each time period with the error estimate expressed as the standard error of the mean during that time period.

\subsection{Spectrometer readings}

To provide independent measures of PRI and NDVI, spectral reflectance was calculated from measurements made by a dual-detector spectrometers (UniSpec DC, PP Systems, Amesbury, MA, USA). For the downward-looking (radiance) detector, the spectrometer foreoptics consisted of a fiber optic (Uni-684, PP Systems, Amesbury, MA, USA) with a FOV restrictor (Hypo-Tube, PP Systems, Amesbury, MA, USA). This yielded a nominal $20^{\circ} \mathrm{FOV}$, but our measurements of the actual FOV yielded estimates closer to $15^{\circ}$, providing a smaller view of the canopy than the SRS sensors having a $20^{\circ}$ 
FOV. For the upward-looking (irradiance) detector, the spectrometer foreoptics consisted of a similar fiber optic (Uni686, PP Systems, Amesbury, MA, USA) with a cosine head (Uni-435, PP Systems, Amesbury, MA, USA). For seasonal studies, 12 canopy reflectance spectra were sampled over each plant stand near solar noon at the same height as the SRS sensors, covering the canopy regions sampled by the SRS sensors, and these 12 scans were expressed as averages $( \pm$ SEM).

Reflectance was calculated by referencing the downwardlooking (radiance) detector to the upward-looking (irradiance) detector (calculating uncorrected reflectance) and then correcting this ratio by means of a cross-calibration procedure using panel measurements similar to that described above (see Gamon et al., 2006, for further details). A standard reference panel (Spectralon, LabSphere, North Sutton, $\mathrm{NH}$, USA) was used as the reference for all reflectance and cross-calibration calculations. To facilitate comparison with the index values of the SRS sensors, identical wavelengths were selected (532 and $570 \mathrm{~nm}$ for PRI, and 630 and 800 for NDVI).

\subsection{Leaf reflectance}

The PRI and NDVI were also measured at the leaf level using a spectrometer (UniSpec SC, PP Systems, Amesbury, MA, USA) configured for leaf reflectance measurements. In this configuration, the spectrometer foreoptics consisted of a bifurcated fiber optic (UNI-410, PP Systems, Amesbury, MA, USA) equipped with a needle leaf clip (UNI-501, PP Systems, Amesbury, MA, USA) to hold the fiber tip at a fixed angle and position relative to the leaf surface. Six plants were randomly selected for leaf reflectance. Five random leaves per plant from the illuminated upper-canopy regions were measured weekly near 13:00 LT (solar noon). Dark measurements and white reference scans (Spectralon, LabSphere, North Sutton, NH, USA) were taken before each sample.

\subsection{Pigment assays}

For pigment assays, leaf tissue samples were collected periodically over the course of the study, during midday (for seasonal experiments), and over the course of a single day (for diurnal experiments). For seasonal studies, leaf samples were collected from the same six plants as the leaf reflectance and $1 \mathrm{~cm}$ long segments from each of the six plants were pooled together for each date and analyzed as a single average. For diurnal studies, four plants from the corners of the plot were selected and two leaves from each plant were obtained in $3 \mathrm{~cm}$ long segments and analyzed as a single average $( \pm$ SEM).

For seasonal studies, needle segments were excised within $30 \mathrm{~min}$ of leaf reflectance (Sect. 2.5), measured with calipers for diameter, and stored in liquid nitrogen until transferred to $\mathrm{a}-80^{\circ} \mathrm{C}$ freezer for long-term storage. For diurnal stud- ies, needles were frozen within a minute of leaf reflectance. To estimate sample area, each segment length was multiplied by the diameter and analyzed with high-performance liquid chromatography (HPLC, 1260 Infinity, Agilent Technologies, Santa Clara, CA, USA). To quantify the concentrations of various carotenoid and chlorophyll pigments, we used the method of Thayer and Björkman (1990). The chlorophyll: carotenoid ratio was calculated as the sum of chlorophyll $a$ and $b$ divided by the sum of all carotenoids including neoxanthin, violaxanthin (V), antheraxanthin (A), lutein, zeaxanthin (Z), and $\beta$-carotene. The epoxidation state (EPS), a measure of xanthophyll cycle activity, was calculated as

$\mathrm{EPS}=\frac{\mathrm{V}+0.5 \mathrm{~A}}{\mathrm{~V}+\mathrm{A}+\mathrm{Z}}$,

where $\mathrm{V}, \mathrm{A}$, and $\mathrm{Z}$ represent the area-based concentrations of violaxanthin, antheraxanthin, and zeaxanthin, respectively.

\section{Results}

\subsection{Cross-calibrations}

For each sensor, cross-calibration ratios varied with sun visibility, which ranged from near-zero under heavy cloud conditions to approximately one under sunny conditions (Fig. 1). In cases of sunny conditions with cumulus clouds, sun visibility values typically exceeded one due to the additional skylight reflected from clouds. On average, cross-calibration ratios approximated the theoretical expectation for radiance / irradiance values of $1 / \pi$, or 0.318 (Monteith, 1973), but clearly varied with sky conditions. Typically, the resulting cross-calibration ratios were higher during sunny than during cloudy conditions and exhibited strong linear relationships with sun visibility (Fig. 1). From these responses, we derived an empirical equation for each sensor band, enabling automatic correction of the midday PRI and NDVI values. These equations were subsequently applied to all midday index calculations involving seasonal trends.

\subsection{Seasonal trends: NDVI and PRI during spring recovery}

The NDVI and PRI were monitored during spring photosynthetic recovery for both species, illustrating the complementary nature of these two indices (Fig. 2). During this time, air temperature increased from a daily average of approximately $0{ }^{\circ} \mathrm{C}$ in late April to approximately $15^{\circ} \mathrm{C}$ by early June (not shown). In $P$. contorta (lodgepole pine), PRI showed an initial increase coincident with a period of increasing chlorophyll: carotenoid ratios and photosynthetic activity (Wong and Gamon, 2015a, b). On the other hand, in the P. tremuloides (aspen) canopy, PRI for was relatively flat, with the exception of a slight rise in canopy-level PRI in May during leaf expansion, followed by a slight decline toward mid-June as leaves matured. This pattern of PRI rise and fall was more 

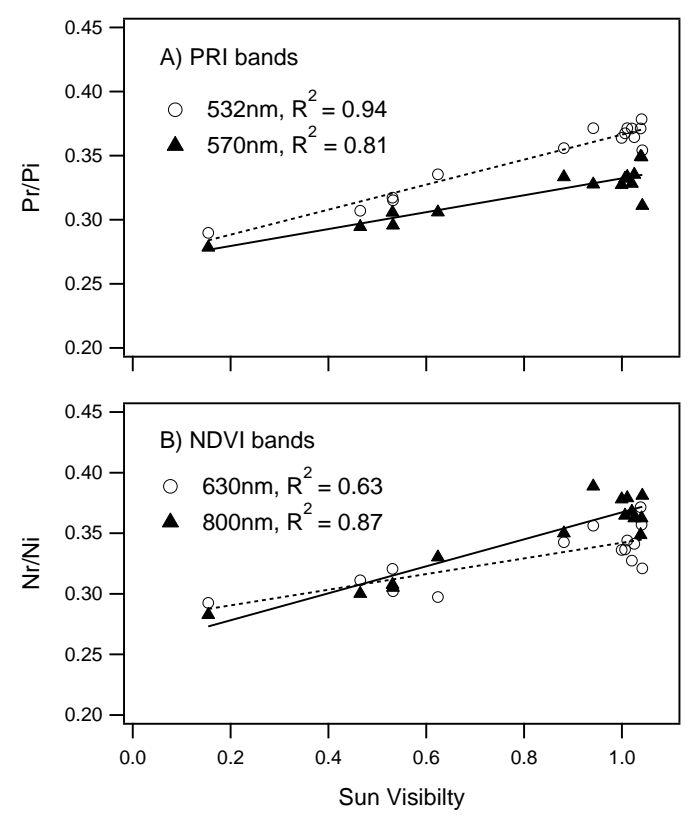

Figure 1. Representative cross-calibration ratios for a single sensor set as a function of sun visibility ratios for PRI bands $(\mathrm{Pr} / \mathrm{Pi}, \mathbf{a})$ and NDVI bands $(\mathrm{Nr} / \mathrm{Ni}, \mathbf{b})$. Sun visibility: 0 is darkness and 1 represents clear, sunny skies, with intermediate values indicating varying degrees of cloudiness.

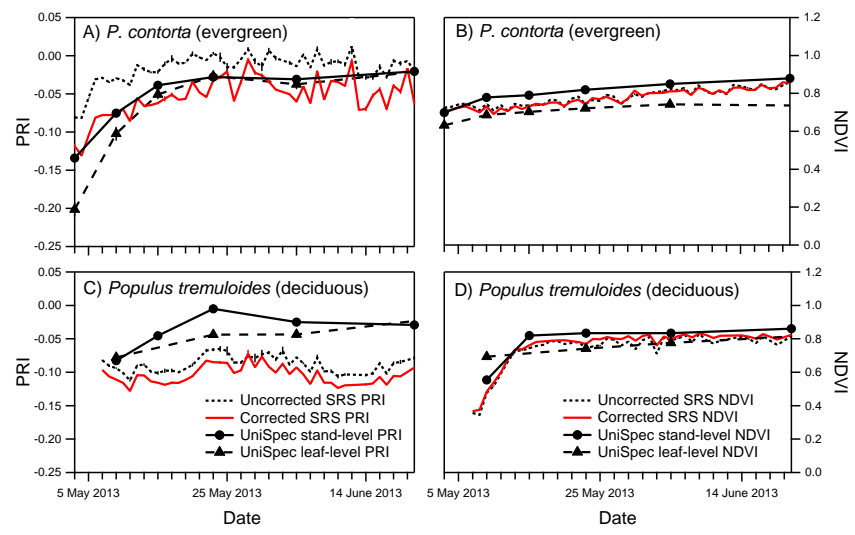

Figure 2. Midday PRI (a and c) and NDVI (b and d) time trends (03 May 2013 to 21 June 2013) for Pinus contorta (lodgepole pine) and Populus tremuloides (trembling aspen) sampled using SRS sensors and spectrometers at both leaf and stand scales. Aspen bud burst began on 05 May, and full expansion was reached on 16 May.

apparent in the spectrometer PRI than in the SRS PRI sensor (Fig. 2c), in part due to the greater short-term dynamics in the SRS values. SRS cross-calibration improved the agreement with spectrometer PRI for $P$. contorta but slightly decreased the agreement for $P$. tremuloides (different sensor pairs were used for each species).

For the pine species, the NDVI trend was nearly flat, but for the aspen stand it showed a marked increase during ini- tial bud burst and leaf expansion. For the pine, these patterns were consistent across instruments and sampling scale (leaf vs. stand). For the aspen, leaf NDVI showed a relatively flat response during the sudden increase in stand NDVI during leaf expansion in early May. Earlier leaf-level sampling was not possible in the aspen because leaves had not yet emerged from the buds. Unlike the effect on PRI, cross-calibration of SRS sensors yielded little change in the SRS NDVI values.

The strong rise in midday PRI for $P$. contorta during spring photosynthetic activation is shown in more detail, along with midday PPFD and pigment trends (Fig. 3). Because the SRS sensors were not available for the early part of this period, PRI values from a spectrometer were added to the plot to show the full period of spring transition associated with photosynthetic activation (for detailed information on these spring physiological changes, see Wong and Gamon, 2015a, b). In this case, cross-calibration of the SRS PRI sensor improved the agreement with spectrometer PRI (Fig. 3a). The spring rise in PRI was coincident with a rise in chlorophyll: carotenoid pigment ratios but not with the xanthophyll cycle epoxidation state (EPS), which increased about 3 weeks earlier than either the PRI or pigment ratios (Fig. 3b). The considerable short-term variability in the SRS PRI signal (particularly visible in late May to early June) was largely attributable to the day-to-day variation in midday PPFD, with PRI declining during sunny days, and rising during cloudy days (Fig. 3a).

\subsection{Diurnal experiments}

Next, we explored the ability of the SRS PRI sensors to resolve diurnal patterns related to xanthophyll cycle activity, as affected by diurnal irradiance. Both xanthophyll cycle EPS and PRI declined towards midday as PPFD increased, and they recovered in the afternoon as PPFD declined (Fig. 4). At very low sun angles, when PPFD values were low and the sun was sometimes partly obscured by objects near the horizon (before 08:00 and after 19:00), PRI values were extremely noisy (indicated by the erratic pattern and large error bars). To test the effect of cross-calibrations on diurnal PRI responses, we first applied midday cross-calibration equations (Fig. 1) using the sun visibility values prevailing during each sample. We also applied empirical cross-calibrations closest in time to each sample, considering both sky conditions and sun angle. The exact PRI pattern was strongly influenced by which of the two cross-calibration methods were applied. The most noticeable effect of the midday cross-calibration was a downward shift in the absolute PRI values, similar to the effect seen in the seasonal PRI patterns for $P$. contorta (Fig. 4c, solid black line). Application of empirical crosscalibrations (using the values closest in time to each sample) further changed the shape of the diurnal PRI pattern, leading to a more pronounced dip and recovery in PRI (Fig. 4c, solid red line) that more closely matched the diurnal pattern of the xanthophyll cycle pigment epoxidation state (EPS) (Fig. 4b). 

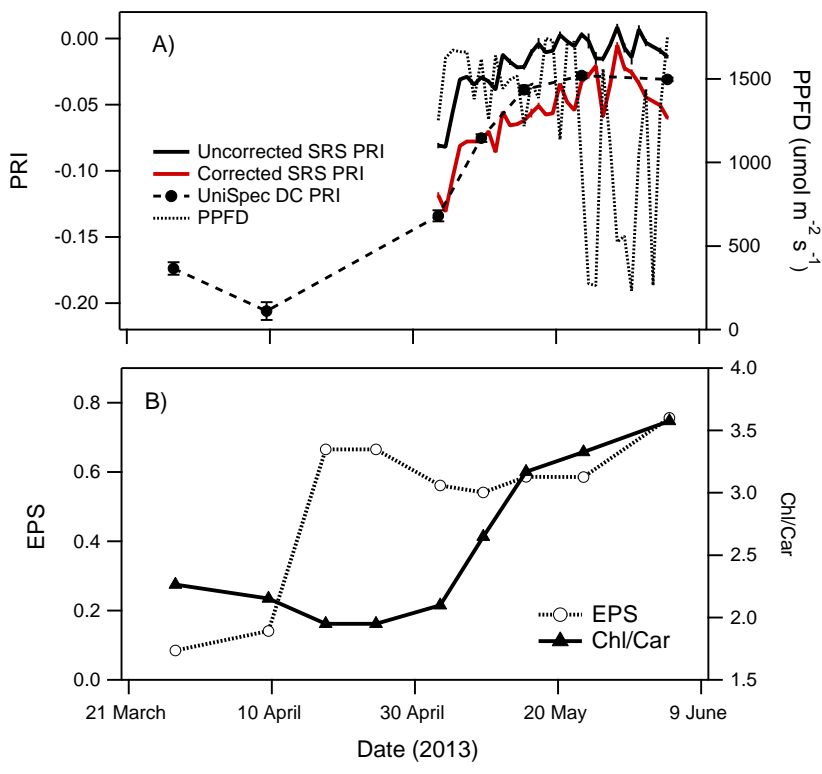

Figure 3. Midday PRI and PPFD trends (a) and pigment trends (b) of $P$. contorta during spring photosynthetic activation. The PRI values were measured by the Decagon SRS sensors and a dualchannel spectrometer (UniSpec DC, PP Systems, Amesbury, MA). Corrected PRI values were produced by applying the empirical midday cross-calibration equations, derived from linear trends (Fig. 1). PRI error bars are standard error of the mean. Chl / Car ratios and xanthophyll cycle epoxidation state (EPS) were single values with no error bars (see Methods section).

\subsection{Comparing PRI to pigments over diurnal and seasonal timescales}

To evaluate the cause of PRI variation over diurnal and seasonal time periods, PRI values recorded by the SRS sensors were compared to pigment data (chlorophyll : carotenoid ratios and xanthophyll cycle epoxidation state). Seasonal measurements spanned the period of spring recovery of photosynthesis in $P$. contorta (03 May 2013 to 04 June 2013) (Fig. 3) and diurnal measurements were collected from a single experiment on 25 July 2013 (Fig. 4).

These comparisons illustrated that, over the seasonal timescale, PRI was correlated with the chlorophyll : carotenoid pigment ratios, but not with the xanthophyll cycle EPS (Fig. 5a and b). Time trends showed that spring recovery of EPS occurs 3-4 weeks before the increase in chlorophyll : carotenoid pigment ratios (Fig. 3), and it is these pigment ratios (not EPS) that best corresponded to the spring increase in PRI (Fig. 5). However, the reverse was true over the diurnal timescale, when PRI was clearly correlated with the xanthophyll cycle EPS (Figs. 4 and 5d), and not the chlorophyll: carotenoid pigment ratios (Figs. 4 and 5c). This result was consistent with the similar diurnal patterns of both PRI and EPS, combined with the relatively flat diurnal pattern of the pigment pool ratios (Fig. 4b).

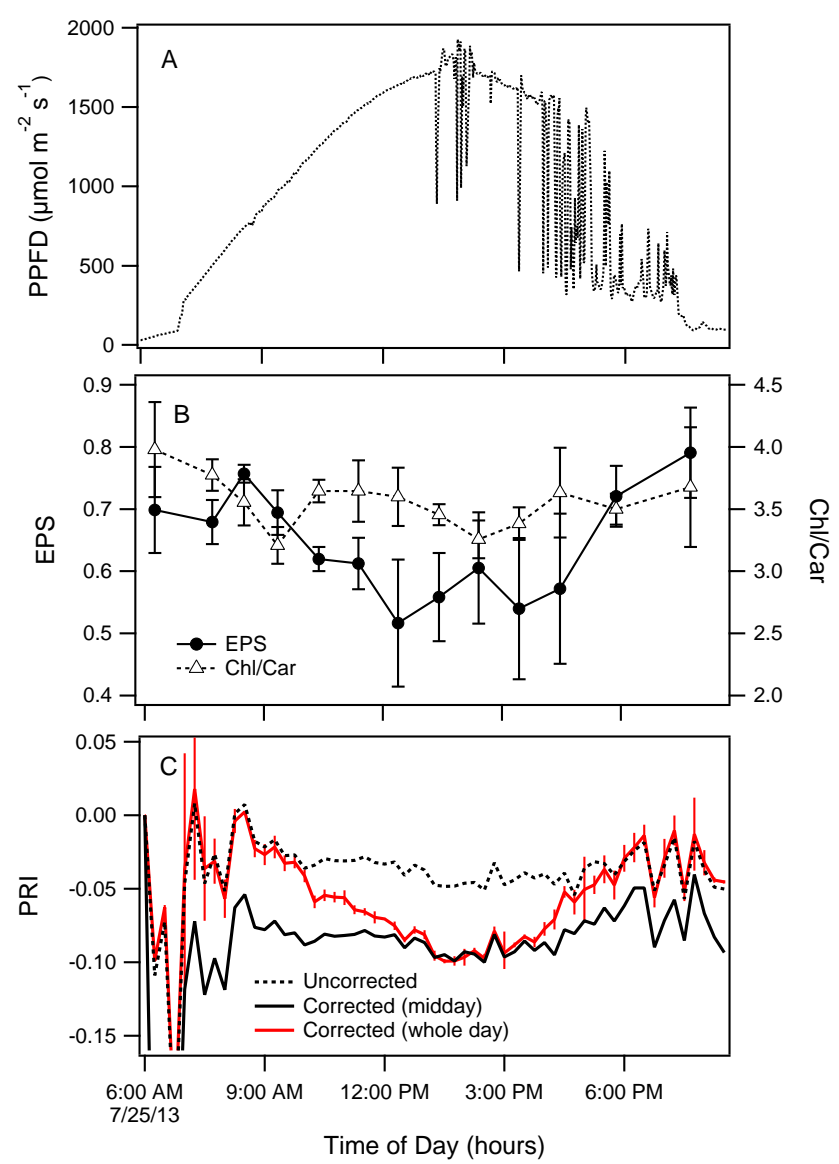

Figure 4. (a) PAR irradiance (PPFD) over the course of the day (25 July 2013). (b) pigment values (epoxidation state and chlorophyll : carotenoid pigment ratios) over the course of the day. (c) Uncorrected and corrected PRI values plotted at $15 \mathrm{~min}$ intervals over the course of the day. Uncorrected PRI values were calculated using Eq. (2). Corrected PRI data (midday correction) were calculated by applying the empirical midday cross-calibrations (Fig. 1), where the sun visibility (cloud cover) is taken into consideration but not the sun angle. Corrected PRI data (whole-day correction) were calculated using the hourly white panel ( $\mathrm{Pr} / \mathrm{Pi}$ ) ratios obtained throughout the day, using the ratio nearest in time. Error bars are \pm 1 SEM for EPS, Chl / Car, and PRI. For clarity, only the error bars for the corrected (whole-day) PRI are shown.

\section{Discussion}

As expected, NDVI and PRI showed complementary behavior in evergreen and deciduous canopies in early spring. In this sense, evergreens and deciduous species represent distinct optical types, as revealed by their contrasting NDVI and PRI behavior. Stand-level NDVI increased in deciduous canopies during leaf emergence and expansion in early spring, but not in evergreen canopies that did not add new leaves during this period. By contrast, PRI detected the changing pigment pool sizes during spring in the evergreen stands, and showed relatively little change in the decidu- 

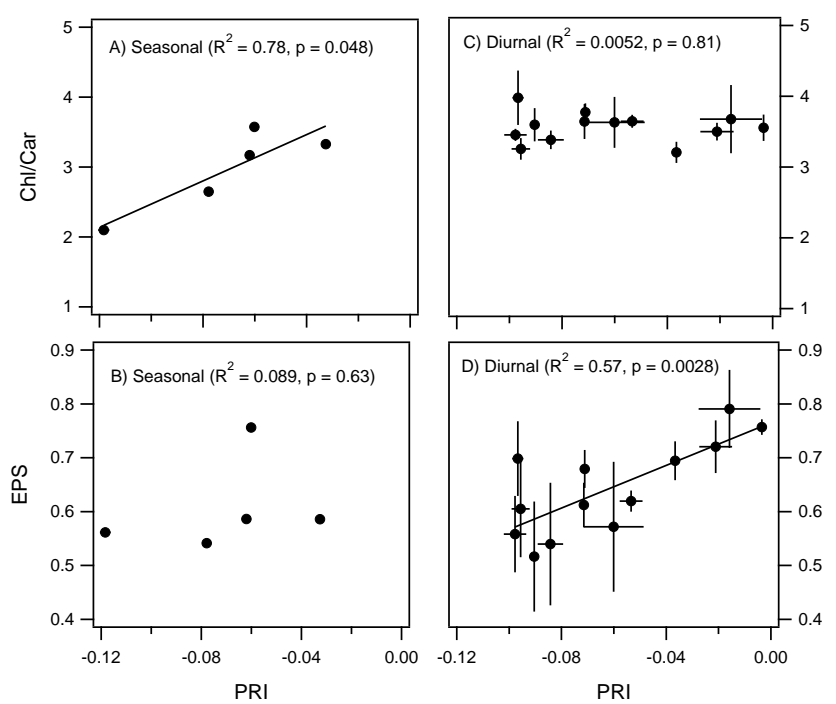

Figure 5. Corrected PRI vs. pigment measures (xanthophyll cycle epoxidation state, EPS, or chlorophyll : carotenoid ratio, Chl / Car). Seasonal data (a, b) span the spring recovery period (03 May 2013 to 04 June 2013; see Fig. 3). Diurnal data (c, d) are from 25 July 2013 (see Fig. 4). Error bars indicate \pm SEM for PRI and diurnal pigment data. Linear regressions are shown for significant $(p<0.05)$ fits only.

ous stands during this period. These complementary patterns emerged both with the SRS sensors and with the field spectrometer, and they demonstrate that automated NDVI and PRI sensors can provide useful information on the contrasting photosynthetic phenology of evergreen and deciduous species. What is particularly intriguing is the ability of PRI to detect changing chlorophyll : carotenoid ratios in the evergreen pine stands. Recent work (Wong and Gamon, 2015, a, b) has shown that these changing pigments (and hence PRI) can provide an indicator of evergreen spring physiological activation, a process that is hard to detect with the eye or with conventional optical remote sensing. On the other hand, NDVI readily captures the changing photosynthetic capacity associated with bud burst and leaf development, but not the less visible evergreen pigment changes during spring. Based on these findings, we would expect that ecosystems from different biomes with contrasting evergreen and deciduous stand composition would show contrasting behavior of NDVI and PRI (Garbulsky et al., 2011). Due to the lack of suitable sensors, this hypothesis has been hard to test extensively, and the advent of automated NDVI and PRI sensors could now enable such comparative tests across contrasting ecosystems. Since these two indices relate to the two terms of the light-use efficiency model (Gamon and Qiu, 1999), better understanding of their complementary behavior could help improve the application of the light-use efficiency model for prediction of ecosystem photosynthesis.

In the evergreen lodgepole pine stand, the SRS PRI was clearly affected by two different processes operating over dif- ferent timescales. Over the diurnal timescale, the SRS PRI followed the changing xanthophyll cycle epoxidation state (EPS), as has been reported before (Gamon et al., 1992). Over a longer time period of several weeks, the midday PRI values followed the changing chlorophyll: carotenoid pool sizes associated with spring photosynthetic activation. Thus, PRI provided a sensitive indicator of pigment changes associated with photosynthetic activity, but in different ways and with different mechanisms. These contrasting mechanisms have been termed the "facultative" (diurnal xanthophyll activity) and "constitutive" (longer-term pigment pool size shifts) responses (Gamon and Berry, 2012; Wong and Gamon, 2015a). Recently, Wong and Gamon (2015a) reported similar findings for evergreens; during seasonal transitions, the PRI signal primarily reflects changing pigment pool sizes, while over the course of a day, PRI detects the diurnal activity of the xanthophyll cycle. This finding is relevant to remote sensing studies that use PRI as an indicator of photosynthetic activity or light-use efficiency, and indicates that the meaning of PRI changes with the temporal context of the study. For most remote sensing studies that rely on a single, midday overpass, it is more likely that pigment pool sizes changes, and not xanthophyll cycle activity, are the primary drivers of PRI changes. Consequently, the interpretation of PRI in a remote sensing context should be re-thought to consider the constitutive response of pigment pools. The advent of automated sensors capable of resolving these two causes of PRI variation in time could be very useful in validating interpretations of PRI detected by airborne and satellite sensors.

The similar responses of PRI observed on a leaf and stand scale (Fig. 2) agree with several previous studies showing parallel leaf- and stand-level PRI responses, at least for closed-canopy stands (Stylinski et al., 2002; Gamon and Qiu, 1999; Wong and Gamon, 2015b). This parallel behavior indicates that dynamic leaf PRI responses are clearly detectable at larger stand scales. Recently, there has been some controversy over whether leaf optical traits are indeed detectable at larger scales with remote sensing, with some authors suggesting that the dominant effect of canopy structure renders leaf traits difficult to detect (Knyazikhin et al., 2013). In response, Townsend et al. (2013) argued that leaf traits are indeed remotely detectable, but that canopy structure can confound the interpretation of such traits. While our findings of parallel leaf- and canopy-level PRI responses suggest that leaf traits are detectable at stand scales, the slightly different patterns emerging from the two contrasting species with contrasting canopy structures suggest that canopy structure may indeed confound these signals to some degree, as has been predicted before for PRI (Barton and North, 2001). It is likely that the interpretation of the PRI becomes far more difficult in mixed stands with both evergreen and deciduous species, or in landscapes with varying degrees of canopy cover. However, at least for closed-canopy monocultures, the temporal patterns of PRI are clearly detectable at both leaf and canopy 
scales, offering some promise for plans to apply PRI to photosynthetic estimation from space (Grace et al., 2007; Coops et al., 2010).

Our findings demonstrate the importance of proper crosscalibration when applying dual-detector sensors, and illustrate that such calibrations should consider the full range of sky conditions encountered. The reason for this is that sensor detector and foreoptics are never perfectly matched, and this matching changes slightly with sky conditions, as has previously been reported (Gamon et al., 2006). We suggest that proper cross-calibration is essential to obtaining correct index values, particularly if these values are to be compared across sensor pairs, sky conditions, or sun angles (e.g., time of day or latitude).

Our results demonstrated clear effects of cross-calibration on the resulting index values, and these effects were more apparent for PRI than for NDVI. The two cross-calibration methods (midday and diurnal) had different effects. The midday method primarily corrected for changing cloud cover, but had little effect on the diurnal PRI patterns. The diurnal method also corrected for diurnally changing sun angle, and this yielded better agreement with diurnal xanthophyll cycle activity (EPS). These results indicate that the recommended method of cross-calibration would vary depending upon the particular purpose. For observing seasonal trends with midday measurements, the midday cross-calibration method may be sufficient. This method yielded predictable results across a wide range of sky conditions (cloudy to clear), which allowed us to model the response with a linear equation. Because it can be modeled, this approach enables automated correction for sky conditions (direct vs. diffuse radiation), which would be of benefit in situations where manual correction is difficult. Automated correction is particularly desirable for automated, remote applications (e.g., tower-mounted applications at remote sites), where frequent manual crosscalibrations may be impossible.

The diurnal cross-calibrations had the benefit of correcting for both sky conditions (clouds) and sun angle, yielding PRI values that better tracked the diurnal changes in xanthophyll cycle EPS. However, this method required frequent manual sampling of a calibration standard over the course of a day. In our study, this method did not yield a single, predictable equation (not shown), so it is unlikely to be easily automated. The reason for this is most likely due to the combined, interacting effects of sun angle (a relatively predictable phenomenon) with changing sky conditions (a less predictable phenomenon). Consequently, the accurate estimation of diurnally changing PRI signals with the SRS sensors, while possible with intensive manual calibrations, is a topic deserving further study.

In the pine stand, cross-calibration clearly improved agreement between SRS and spectrometer PRI values, but not in the aspen stand. The underlying reasons for this difference are not entirely clear, but we suspect that it may result from the contrasting canopy structures combined with the different sensor FOVs. Aspen leaves have a strong vertical orientation, and it is likely that this caused the narrow FOV spectrometer to "look deeper" into the canopy, thus having a greater contribution of shaded (high PRI) leaves to the overall stand PRI signal. On the other hand, the broader FOV SRS sensors presumably detected a higher proportion of sunlit leaves high in the canopy causing a lower canopy PRI value. This hypothesis may explain why cross-calibration did not improve the agreement between SRS and spectrometer PRI measurements for the aspen canopy. On the other hand, in the pine, the leaf angles were more randomly distributed, and this may have allowed a much better agreement between SRS and spectrometer PRI values following cross-calibration. In the case of the pine canopy, cross-calibration clearly led to closer agreement of the SRS and spectrometer PRI values.

Our results agree with previous findings that sun-canopysensor geometry can exert a strong effect on the resulting index values (Sims et al., 2006; Hilker et al., 2008). In many canopies, light fields vary in complex ways with canopy structure and aspect, causing strong differences between optical measurements made from slightly different positions, even within a single stand (Middleton et al., 2009; Gamon and Bond, 2013). For this reason, studies using proximal sensors over canopies with complex light fields should carefully consider the canopy structure and illumination regime, and select sensor distance and sampling angle accordingly. While not a central part of our study due to the limited number of sensors, the role of sensor position and sampling angle should be investigated in further studies, as well as the required replication needed to obtain representative samples of stand optical properties. This becomes particularly critical if the goal is to relate proximal optical sampling to larger footprints, as is often the case when validating satellite measurements or comparing to flux tower measurements.

What was missing from our short-term study was a full consideration of long-term sensor stability. Temperature stability and the ability to withstand moisture are key considerations, particularly if sensors are to be useful over one or more annual cycles, and these factors were not fully considered in our study. Since completion of the study, the manufacturer (Decagon Devices) has changed the NDVI sensor from the LED version used in this study to a photodiode design in order to attain greater temperature stability. We recommend that additional studies be conducted over a range of environments to more fully test the behavior, utility, stability, and longevity of the SRS sensors. For such studies, the crosscalibration methods described here could be essential, not just for obtaining accurate index values but also for checking and correcting for sensor drift and enabling proper comparison of values across sites. Ideally, such tests would include ecosystems and biomes with contrasting optical behavior and environmental constraints on photosynthesis, allowing us to more fully develop the concept of optical types. Our hope is that the initial findings reported here can provide a first step in developing protocols for such a study. 


\section{Conclusions}

PRI and NDVI detected complementary processes during spring transition in evergreen and deciduous canopies. As expected, NDVI was primarily sensitive to leaf emergence in deciduous aspen stands, and PRI was sensitive to changing pigment ratios in evergreen pine stands. PRI was also able to detect diurnal changes in xanthophyll cycle epoxidation state, although the primary cause of PRI increase during spring was the increasing chlorophyll: carotenoid ratio, and not the xanthophyll cycle.

The diurnal and seasonal patterns were clearly sensitive to the method of cross-calibration. For each sensor, sun visibility (cloud cover) had a predictable effect on the crosscalibration, allowing us to model this correction for each sensor. Determining this response for each sensor should facilitate automated application of optical sensors where regular calibration would not be feasible. On the other hand, due to the combined effects of sun angle and sky conditions, obtaining accurate diurnal responses may require frequent manual calibration that may present challenges for sensor automation.

Automated, low-cost NDVI and PRI sensors offer new opportunities for monitoring photosynthetic phenology. We recommend further tests be applied over longer time periods at flux tower sites across a range of ecosystems, with a particular focus on the optical responses of contrasting vegetation types. Such studies would help improve our understanding of the component terms of the light-use efficiency model and could help reveal contrasting controls on carbon flux for different ecosystems.

Acknowledgements. We thank Steve Williams, Morgan Randall, and Saulo Castro for assistance in plant care, and Enrica Nestola for expert help during the diurnal experiment. Funding to J. A. Gamon for this work was provided by the NSERC Discovery, iCOREAITF, and CFI programs. We also thank Decagon Devices for the generous loan of SRS sensors, making this study possible. Travel support for Enrica Nestola and Angela Harris was provided by a EUROSPEC - Cost Action ES0903 Short Term Scientific Mission grant.

Edited by: E. Tomelleri

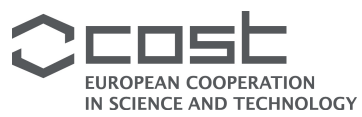

This publication was supported by COST - www.cost.eu

\section{References}

Adams, W. W., Demmig-Adams, B., Rosenstiel, T. N., Brightwell, A. K., and Ebbert, V.: Photosynthesis and photoprotection in overwintering plants, Plant Biol., 4, 545-557, doi:10.1055/s2002-35434, 2002.

Barton, C. V. M. and North, P. R. J.: Remote sensing of canopy light use efficiency using the photochemical reflectance index -

Model and sensitivity analysis, Remote Sens. Environ., 78, 264 273, doi:10.1016/s0034-4257(01)00224-3, 2001.

Castro-Esau, K. L., Sanchez-Azofeifa, G. A., and Rivard, B.: Comparison of spectral indices obtained using multiple spectroradiometers, Remote Sens. Environ., 103, 276-288, doi:10.1016/j.rse.2005.01.019, 2006.

Coops, N. C., Hilker, T., Hall, F. G., Nichol, C. J., and Drolet, G. G.: Estimation of light-use efficiency of terrestrial ecosystem from space: A status report, Bioscience, 60, 788-797, doi:10.1525/bio.2010.60.10.5, 2010.

DeFries, R. S. and Townshend, J. R. G.: NDVI-derived land-cover classifications at a global-scale, Int. J. Remote Sens., 15, 35673586, 1994.

Demmig-Adams, B. and Adams, W. W.: Photoprotection and other responses of plants to high light stress, Annu. Rev. Plant Phys., 43, 599-626, doi:10.1146/annurev.pp.43.060192.003123, 1992.

Drolet, G., Wade, T., Nichol, C. J., MacLellan, C., Levula, J., Porcar-Castell, A., Nikinmaa, E., and Vesala, T.: A temperaturecontrolled spectrometer system for continuous and unattended measurements of canopy spectral radiance and reflectance, Int. J. Remote Sens., 35, 1769-1785, 2014.

Eklundh, L., Jin, H., Schubert, P., Guzinski, R., and Heliasz, M.: An optical sensor network for vegetation phenology monitoring and satellite data calibration, Sensors, 11, 7678-7709, doi:10.3390/s110807678, 2011.

Filella, I., Porcar-Castell, A., Munne-Bosch, S., Back, J., Garbulsky, M. F., and Peñuelas, J.: PRI assessment of long-term changes in carotenoids/chlorophyll ratio and short-term changes in deepoxidation state of the xanthophyll cycle, Int. J. Remote Sens., 30, 4443-4455, doi:10.1080/01431160802575661, 2009.

Gamon, J. and Qiu, H.-L.: Ecological applications of remote sensing at multiple scales, in: Handbook of Functional Plant Ecology, edited by: Pugnaire, F. and Valladares, F., Marcel Dekker, Inc., New York, 805-846, 1999.

Gamon, J. A. and Berry, J. A.: Facultative and constitutive pigment effects on the Photochemical Reflectance Index (PRI) in sun and shade conifer needles, Isr. J. Plant Sci., 60, 85-95, doi:10.1560/ijps.60.1-2.85, 2012.

Gamon, J. A. and Bond, B.: Effects of irradiance and photosynthetic downregulation on the photochemical reflectance index in Douglas-fir and ponderosa pine, Remote Sens. Environ., 135, 141-149, doi:10.1016/j.rse.2013.03.032, 2013.

Gamon, J. A., Peñuelas, J., and Field, C. B.: A narrow-waveband spectral index that tracks diurnal changes in photosynthetic efficiency, Remote Sens. Environ., 41, 35-44, doi:10.1016/00344257(92)90059-s, 1992.

Gamon, J. A., Field, C. B., Goulden, M. L., Griffin, K. L., Hartley, A. E., Joel, G., Peñuelas, J., and Valentini, R.: Relationships between NDVI, canopy structure, and photosynthesis in three Californian vegetation types, Ecol. Appl., 5, 28-41, doi:10.2307/1942049, 1995.

Gamon, J. A., Serrano, L., and Surfus, J. S.: The photochemical reflectance index: an optical indicator of photosynthetic radiation use efficiency across species, functional types, and nutrient levels, Oecologia, 112, 492-501, doi:10.1007/s004420050337, 1997.

Gamon, J. A., Field, C. B., Fredeen, A. L., and Thayer, S.: Assessing photosynthetic downregulation in sunflower stands 
with an optically-based model, Photosynth. Res., 67, 113-125, doi:10.1023/a:1010677605091, 2001.

Gamon, J. A., Cheng, Y. F., Claudio, H., MacKinney, L., and Sims, D. A.: A mobile tram system for systematic sampling of ecosystem optical properties, Remote Sens. Environ., 103, 246-254, doi:10.1016/j.rse.2006.04.006, 2006.

Garbulsky, M. F., Peñuelas, J., Gamon, J., Inoue, Y., and Filella, I.: The photochemical reflectance index (PRI) and the remote sensing of leaf, canopy and ecosystem radiation use efficiencies A review and meta-analysis, Remote Sens. Environ., 115, 281-297, doi:10.1016/j.rse.2010.08.023, 2011.

Garrity, S. R., Vierling, L. A., and Bickford, K.: A simple filtered photodiode instrument for continuous measurement of narrowband NDVI and PRI over vegetated canopies, Agr. Forest Meteorol., 150, 489-496, doi:10.1016/j.agrformet.2010.01.004, 2010.

Garrity, S. R., Eitel, J. U. H., and Vierling, L. A.: Disentangling the relationships between plant pigments and the photochemical reflectance index reveals a new approach for remote estimation of carotenoid content, Remote Sens. Environ., 115, 628-635, doi:10.1016/j.rse.2010.10.007, 2011.

Goward, S. N., Tucker, C. J., and Dye, D. G.: NorthAmerican vegetation patterns observed with the NOAA-7 Advanced Very High-Resolution Radiometer, Vegetatio, 64, 3-14, doi:10.1007/bf00033449, 1985.

Grace, J., Nichol, C., Disney, M., Lewis, P., Quaife, T., and Bowyer, P.: Can we measure terrestrial photosynthesis from space directly, using spectral reflectance and fluorescence?, Glob. Change Biol., 13, 1484-1497, doi:10.1111/j.13652486.2007.01352.x, 2007.

Harris, A., Gamon, J. A., Pastorello, G. Z., and Wong, C. Y. S.: Retrieval of the photochemical reflectance index for assessing xanthophyll cycle activity: a comparison of near-surface optical sensors, Biogeosciences, 11, 6277-6292, doi:10.5194/bg-11-62772014, 2014.

Hilker, T., Coops, N.C., Nesic, Z., Wulder, M. A., and Black, A. T.: Instrumentation and approach for unattended year round tower based measurements of spectral reflectance, Comput. Electron. Agr., 56, 72-84, doi:10.1016/j.compag.2007.01.003, 2007.

Hilker, T., Coops, N. C., Hall, F. G., Black, T. A., Wulder, M. A., Nesic, Z., and Krishnan, P.: Separating physiologically and directionally induced changes in PRI using BRDF models, Remote Sens. Environ., 112, 2777-2788, doi:10.1016/j.rse.2008.01.011, 2008.

Knyazikhin, Y., Schull, M. A., Stenberg, P., Mottus, M., Rautiainen, M., Yang, Y., Marshak, A., Latorre Carmona, P., Kaufmann, R. K., Lewis, P., Disney, M. I., Vanderbilt, V., Davis, A. B., Baret, F., Jacquemoud, S., Lyapustin, A., and Myneni, R. B.: Hyperspectral remote sensing of foliar nitrogen content, P. Natl Acad. Sci. USA, 110, E185-E192, doi:10.1073/pnas.1210196109, 2013.

Middleton, E. M., Cheng, Y.-B., Hilker, T., Black, T. A., Krishnan, P., Coops, N. C., and Huemmrich, K. F.: Linking foliage spectral responses to canopy-level ecosystem photosynthetic light-use efficiency at a Douglas-fir forest in Canada, Can. J. Remote Sens., 35, 166-188, 2009.

Monteith, J. L.: Principles of Environmental Physics, Elsevier, New York, 1973.
Peñuelas, J., Filella, I., and Gamon, J. A.: Assessment of photosynthetic radiation-use efficiency with spectral reflectance, New Phytol., 131, 291-296, doi:10.1111/j.14698137.1995.tb03064.x, 1995.

Porcar-Castell, A., Ignacio Garcia-Plazaola, J., Nichol, C. J., Kolari, P., Olascoaga, B., Kuusinen, N., Fernandez-Marin, B., Pulkkinen, M., Juurola, E., and Nikinmaa, E.: Physiology of the seasonal relationship between the photochemical reflectance index and photosynthetic light use efficiency, Oecologia, 170, 313323, doi:10.1007/s00442-012-2317-9, 2012.

Rossini, M., Cogliati, S., Meroni, M., Migliavacca, M., Galvagno, M., Busetto, L., Cremonese, E., Julitta, T., Siniscalco, C., Morra di Cella, U., and Colombo, R.: Remote sensing-based estimation of gross primary production in a subalpine grassland, Biogeosciences, 9, 2565-2584, doi:10.5194/bg-9-2565-2012, 2012.

Running, S. W., Nemani, R. R., Heinsch, F. A., Zhao, M. S., Reeves, M., and Hashimoto, H.: A continuous satellite-derived measure of global terrestrial primary production, Bioscience, 54, 547560, doi:10.1641/0006-3568(2004)054[0547:acsmog]2.0.co;2, 2004.

Ryu, Y., Baldocchi, D. D., Verfaillie, J., Ma, S., Falk, M., RuizMercado, I., Hehn, T., and Sonnentag, O.: Testing the performance of a novel spectral reflectance sensor, built with light emitting diodes (LEDs), to monitor ecosystem metabolism, structure and function, Agr. Forest Meteorol., 150, 1597-1606, doi:10.1016/j.agrformet.2010.08.009, 2010.

Sims, D. A. and Gamon, J. A.: Relationships between leaf pigment content and spectral reflectance across a wide range of species, leaf structures and developmental stages, Remote Sens. of Environ., 81, 337-354, doi:10.1016/s0034-4257(02)00010-x, 2002.

Sims, D. A., Luo, H. Y., Hastings, S., Oechel, W. C., Rahman, A. F., and Gamon, J. A.: Parallel adjustments in vegetation greenness and ecosystem $\mathrm{CO}_{2}$ exchange in response to drought in a Southern California chaparral ecosystem, Remote Sens. of Environ., 103, 289-303, doi:10.1016/j.rse.2005.01.020, 2006.

Stylinski, C. D., Gamon, J. A., and Oechel, W. C.: Seasonal patterns of reflectance indices, carotenoid pigments and photosynthesis of evergreen chaparral species, Oecologia, 131, 366-374, doi:10.1007/s00442-002-0905-9, 2002.

Thayer, S. S. and Björkman, O.: Leaf xanthophyll content and composition in sun and shade determined by HPLC, Photosynth. Res., 23, 331-343, doi:10.1007/bf00034864, 1990.

Townsend, P. A., Serbin, S. P., Kruger, E. L., and Gamon, J. A.: Disentangling the contribution of biological and physical properties of leaves and canopies in imaging spectroscopy data, P. Natl. Acad. Sci. USA, 110, E1074-E1074, doi:10.1073/pnas.1300952110, 2013.

Wong, C. Y. S. and Gamon, J. A.: Three causes of variation in the Photochemical Reflectance Index (PRI) in evergreen conifers, New Phytol., 206, 187-195, doi:10.1111/nph.13159, 2015a.

Wong, C. Y. S. and Gamon, J. A: The Photochemical Reflectance Index (PRI) provides an optical indicator of spring photosynthetic activity in conifers, New Phytol., 206, 196-208, doi:10.1111/nph.13251, 2015b. 